\title{
Interactive Projection Mapping: Proyecto Patrimonio ANGLO Interactive Projection Mapping: ANGLO Heritage Project
}

\author{
Arq. Gabriela Barber \\ Vidialab de Facultad de Arquitectura \\ de la UdelaR, Uruguay \\ gbarber@fadu.edu.uy \\ Lic. Marcos Lafluf \\ Vidialab de Facultad de Arquitectura de \\ la UdelaR, Uruguay \\ marcoslafluf@fadu.edu.uy
}

\begin{abstract}
This approach to the videomapping technique rises from the experience in the Vidialab environment. Starting from the digital fabrication of a representative model of Fray Bentos industrial landscape, declared World Heritage by UNESCO, a videomapping project develops with certain peculiarities. Here we expose this technique applied to a model as a form to explore tools in order to experience the heritage in an enriched and attractive way, combining image, information and immersive environments. Also the procedures used to provide an educational streak are presented.
\end{abstract}

Keywords: Videomapping, Nuevos medios, New media art, Difusión patrimonial, Objetos de aprendizaje.

\section{Introducción}

El presente trabajo se enmarca en el proyecto de investigación "La ciudad inteligente; un palimpsesto digital" en desarrollo en el Laboratorio de Visualización Digital Avanzada (Vidialab) dependiente del Departamento de Informática aplicada al Diseño (Deplnfo) de la Facultad de Arquitectura, Diseño y Urbanismo (UdelaR). Dicho proyecto tiene como motivación la reciente designación del "Paisaje Cultural Industrial Fray Bentos" como patrimonio de la humanidad por la UNESCO en el año 2015.

El Paisaje Industrial Fray Bentos se ubica en el Departamento de Río Negro (Uruguay) y abarca 275 hectáreas que incluyen la arquitectura del frigorífico Liebig's-Anglo, sus instalaciones industriales, los muelles sobre el río Uruguay, el matadero, las áreas dedicadas al pastoreo, las residencias de jefes y obreros y sus lugares de esparcimiento. IMRN (2015). Su valor radica en que el lugar permite "(...) aprehender la totalidad del proceso de una producción de carne que tuvo importancia mundial." 1

A partir de escaneo por fotografías se llegó a un modelo digital tridimensional que se materializó en una maqueta a escala $1 / 400$ hecha con fabricación digital en pvc blanco y cartón por el LabFAB (Laboratorio de Fabricación Digital), dependiente del Deplnfo. Se busca integrar esta maqueta a un entorno de exposición en el lugar de una manera innovadora al incorporar la interacción con el videomapping.
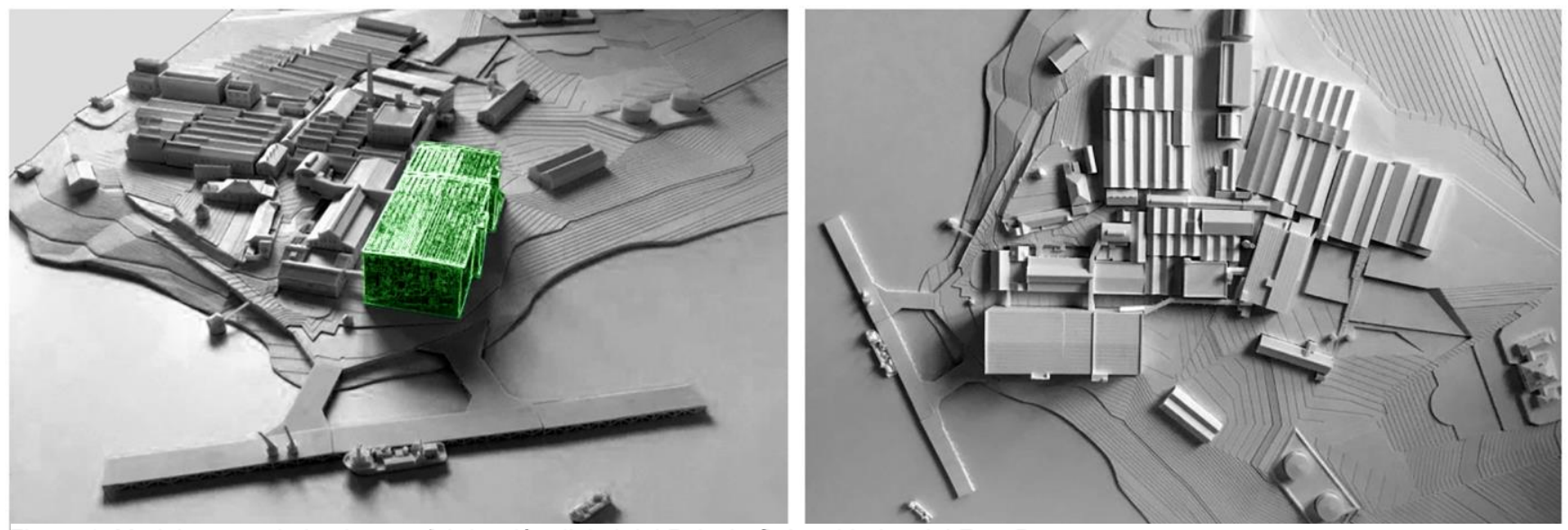

Figura 1. Modelo a escala hecho con fabricación digital del Paisaje Cultural Industrial Fray Bentos

${ }^{1}$ Unesco 2016 Industrial cultural landscape of Fray Bentos included in the world heritage (on line) Disponible en http://www.unesco.org/new/es/office-in-montevideo/about-thisoffice/single- view/news/industrial_cultural_landscape_of_fray_bentos_includ ed_in_the_world_heritage_list/\#.V2tCjbgrKhe 
El proyecto que se expone, pretende transformar esta representación en un punto de acceso a la información sobre el patrimonio ANGLO a modo de posibilitar al usuario una forma de recorrer el paisaje utilizando multimedia digital interactiva.

Entendiendo el videomapping como realidad aumentada tomando como referencia la conceptualización de Azuma (1997), ésta a diferencia de la realidad virtual permite integrar una dimensión digital a lo real. Esto posibilita un aprendizaje más experimental y experiencial sin eliminar la distancia suficiente que permiten los procesos de asimilación e inferencia de conocimiento. El videomapping como realidad aumentada proyectada, permite y facilita pensar en la educación, entendiéndose que este tipo de tecnologías tienen un futuro prometedor en este campo, en tanto permiten encontrar formas didácticas de comprender la realidad, aumentar la posibilidades de asimilación del individuo y permitir los procesos de inferencia que transforman el objeto (modelo a escala) en conocimiento, al hacerlo más contextualizado como señalan varios autores tales como Prendes(2015) o Garnica\&Calderon(2015).

\section{El videomapping y el modelo a escala como metáfora del palimpsesto digital}

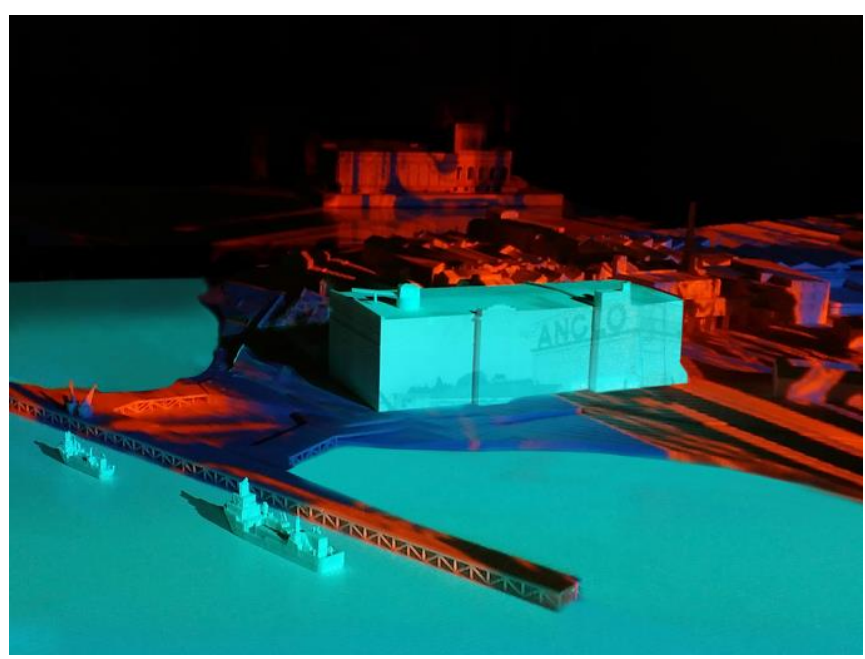

Figura 2. "Mapeado" sobre el modelo a escala

El proyecto I+D "La ciudad inteligente; un palimpsesto digital" propone la incorporación de contenidos digitales en el espacio arquitectónico-urbano en el contexto patrimonial. Dentro de estos contenidos encontramos aplicaciones, apps, que buscan la interacción usuario-realidad física-información digital. Los dispositivos de interacción deberán facilitar una interfaz intuitiva minimizando la complejidad de la tecnología que se aplica.

En la parte del proyecto desarrollada en este artículo la realidad física de base es el modelo a escala (intervenido con un videomapping). Este se transforma en un soporte donde distintas layers de información se superponen, conformando de alguna manera ese palimpsesto digital, esa mezcla de estratos que contienen fragmentos de textos, imágenes, sonidos y discursividades provenientes de distintos tiempos,

\footnotetext{
${ }^{2}$ Según la norma UNE-EN 71361

${ }^{3}$ Según el estándar LOM del IEEE Learning Technology Standards
}

disciplinas y perspectivas por las cuales es posible circular sin salir de la misma maqueta/ palimpsesto / territorio.

\section{La interacción con el patrimonio en la era digital}

Las tecnologías de la información como señala Castells (1995) configuran no solo una nueva forma de producción y economía, sino que intervienen en la relación con el entorno desde la producción de significado, la mediación que generan, interrumpiendo y enriqueciendo la experimentación de lo real.

Esta ubicuidad que se hace presente en la comunicación digital tiene su potencial en lo que refiere a generar mayor conocimiento y motivar el acercamiento al patrimonio tangible de una sociedad, motivando su conocimiento y apropiación.

Las industrias culturales tradicionalmente encargadas de salvaguardar y difundir el patrimonio han abierto nuevos espacios ante el avance de las nuevas tecnologías, generando nuevos vínculos con actores de las nuevas industrias creativas $u$ otros actores de distintos ámbitos universitarios como el que se ha dado en este caso.

Actualmente las instituciones y la difusión del patrimonio reclaman novedosas formas de atraer a un nuevo usuario inmerso en un contexto de tics. Esto implica el aporte de distintas áreas como en el caso presente donde el diseño y la arquitectura digital tienen su espacio.

\section{Metodología y Materiales}

Mediante la observación y análisis de corte etnográficocualitativo se pretende la descripción e identificación de las principales metodologías, estrategias y elementos relevantes que se realizan en el planeamiento y puesta en curso del proyecto anteriormente referido. La metodología elegida, se entendió apropiada para una primera exploración.

Para facilitar la observación y descripción dividimos el proyecto en dos grandes capas. La primera capa refiere a la producción/gestión y edición de contenidos digitales que serán proyectados en el modelo real. La segunda capa describe el software-hardware utilizado y los desarrollos llevados a cabo para la implementación del videomapping interactivo.

Estando el proyecto en curso y desarrollo, no permite aún una exposición acabada y concluyente sobre algunos de los aspectos de éste. Queda pendiente para un futuro abordaje, la observación de la relación e interacción del público del museo con la instalación en situ.

\section{Las Dimensiones del Proyecto}

\section{Capa 1: Gestión de contenidos digitales}

Tomando la perspectiva de los ODE (Objetos digitales educativos).

Un punto a destacar en este proyecto es la exploración del potencial de esta técnica teniendo como orientación las pautas para el desarrollo de objetos digitales educativos de segundo nivel $^{2} \mathrm{u}$ objetos de aprendizaje ${ }^{3}$ (vinculados a temáticas de

Committee (2002) en el que se define como tal a cualquier entidad digital o no, que sea utilizada para el aprendizaje. 
patrimonio arquitectónico) desarrolladas principalmente en el ámbito educativo europeo.

Se buscó conectar esta práctica (videomapping) con el universo de los objetos educativos digitales, especialmente lo referente a las pautas que hacen a los diseños teniendo en cuenta la modularidad, interactividad, usabilidad y reutilización.

De igual manera se señalan los límites de esta asociación o relación que planteamos, en tanto no todas las pautas observadas tenían aplicación para nuestro $\mathrm{caso}^{4}$, ya que no estábamos desarrollando estrictamente un $\mathrm{ODE}^{5}$, sino utilizando los criterios de estos para mejorar la propuesta.

Esta opción que llamamos perspectiva de los ODE o educativa condicionó la estrategia tomada para la gestión de contenidos digitales a utilizar en la proyección, teniendo como fundamento para la elección de esta perspectiva tres elementos observados durante el proceso:

- La escala de la maqueta limitaba y dificultaba la realización de una animación estrictamente visual, así como el despliegue de información directamente sobre los elementos de la misma. Por esta razón es que se opta por darle mayor protagonismo a los contenidos informacionales.

- La cantidad de contenidos digitales se conformaba con objetos (unidades) no estructurados 0 sistematizados, lo cual implicaba una necesaria gestión y la utilización de alguna pauta para esta gestión.

- $\quad$ El entendimiento de que una gestión de contenidos digitales que integre la perspectiva educativa permitirá la producción de capas de información estructurada y organizada que abarquen la complejidad histórica y social del objeto de esta instalación.

A partir de estos factores se planteó priorizar los contenidos digitales sobre la performance, teniendo en cuenta los siguientes elementos:

- La integración de algunos de los contenidos utilizados en las unidades programáticas de primaria y secundaria que abordan la temática del Frigorífico Anglo y su contexto histórico.

La temática del frigorífico es abordaba en relación a la primera y segunda guerra mundial, por lo cual se desarrolló a modo piloto menús interactivos que contenían información textual de las mismas, material fotográfico y audiovisual de dominio público, así como animaciones y remix audiovisuales que contenían imágenes capturadas por drones del Vidialab conectándose con registros fotográficos y audiovisuales de la primera y segunda guerra mundial.

${ }^{4}$ Por ejemplo, no aplica la pauta de integrar alguna forma de evaluación o autoevaluación para constatar que se cumplió con un objetivo educativo puntual en el contexto de esta maqueta, por su carácter más de performance, pero si integrar los objetivos de una unidad programática de liceo o escuela, así como los intereses de los docentes.
- Se integran objetos digitales agrupados según una estructuración modular, que permita su reubicación en otros módulos o propuestas de contenido. Esta estructura modular se realiza teniendo como base el Pre Inventario producido por el Instituto de Historia (IHA) de la Facultad de Arquitectura, Diseño y Urbanismo de la Udelar.

- La asociatividad se hace presente a partir de la utilización de los edificios y estructuras representadas en el modelo a escala como elemento de acceso a temáticas que se asocian a estos hechos históricos. A modo de ejemplificación: Barco en río - transporte de mercancía para abastecer durante la primera y segunda guerra mundial: cocina del mundo.

- Reutilización de objetos digitales manejados en el modelo a escala a través de un repositorio digital.

Con respecto a los pasos desarrollados en esta capa, se pueden distinguir las siguientes instancias:

1) Investigación documental y selección de documentos digitalizados de valor histórico.

Fue fundamental el relacionamiento con las instituciones asociadas del contexto donde estaría la instalación por contener la memoria y documentaciónbasociada a éste. Específicamente el Museo de las Revoluciones y su archivo, así como aquellos trabajos de recopilación e investigación realizados por referentes del tema Frigorífico Anglo.

2) Estructuración y producción de contenidos digitales. En esta etapa se aborda la presentación de información, el guion principal de proyección y el diseño digital.

Se desarrollaron estrategias (actividades en el contexto, presentación del proyecto en comunidad, etc.) y metodologías (entrevistas, conversaciones) para integrar la perspectiva del Museo de la Revolución Industrial, actor fundamental, así como de otros actores del medio tales como docentes y profesionales del diseño vinculados al Anglo. También se consulta a docentes sobre los contenidos y el programa que manejan para abordar el tema Anglo en las aulas.
5 Se entiende que no es un ODE propiamente dicho, en tanto
momentáneamente no está planteado como documento hipertextual
en html accesible por web. Si estamos frente a un objeto de
aprendizaje, en tanto se plantea enseñar algunos puntos estudiados
en secundaria/primaria sobre el anglo. 


\begin{tabular}{|c|c|c|c|c|}
\hline PROCESOS & CAPA INFORMÀTICA & HARDWARE & DESCRIPCIÓN & PRODUCTO \\
\hline $\begin{array}{l}\text { Obtención de modelo } \\
\text { digital }\end{array}$ & $\begin{array}{l}\text { Autodesk } 360 \\
\text { 3ds max }\end{array}$ & $\begin{array}{l}\text { Cámara gopro } 4 \\
\text { Drone Dji Phantom } 4\end{array}$ & $\begin{array}{l}\text { Se obtuvo mediante fotografías } \\
\text { aéreas obtenidas con drones } \\
\text { (realizado inicialmente para la } \\
\text { fabricación del modelo a escala del } \\
\text { lugar), y se cargó posteriormente en } \\
\text { la aplicación Autodesk } 360 \text { y en } 3 \text { ds } \\
\text { Max. }\end{array}$ & Modelo digital del lugar \\
\hline $\begin{array}{l}\text { Producción y edición de } \\
\text { contenidos. } \\
\text { Manipulación del } \\
\text { modelo }\end{array}$ & $\begin{array}{l}\text { 3Ds MAX } \\
\text { Adobe Premiere Pro } \\
\text { Adobe After Effects } \\
\text { Adobe Photoshop }\end{array}$ & & $\begin{array}{l}\text { Modelado digital tridimensional en } \\
\text { base a modificadores. Utilización de } \\
\text { software de edición digital de imagen, } \\
\text { video y sonido. }\end{array}$ & $\begin{array}{l}\text { Clips de video y } \\
\text { sonido. Remix digital. } \\
\text { Representación visual } \\
\text { en base a layers de } \\
\text { información. }\end{array}$ \\
\hline $\begin{array}{l}\text { Instalación Projection } \\
\text { mapping. Testing de } \\
\text { imagen y sonido. }\end{array}$ & Resolume Arena & $\begin{array}{l}\text { Proyectores } 3000 \text { lum } \\
\text { Matrox }\end{array}$ & $\begin{array}{l}\text { Pruebas sobre la maqueta con los } \\
\text { proyectores. Acople a la superficie. } \\
\text { Ajustes y calibración de proyectores. }\end{array}$ & $\begin{array}{l}\text { Instalación de } \\
\text { projection mapping. }\end{array}$ \\
\hline Pruebas de interacción. & Ubi & $\begin{array}{l}\text { Kinect } \\
\text { Proyector }\end{array}$ & $\begin{array}{l}\text { Usando un sensor de movimiento, la } \\
\text { cámara Kinect y software que } \\
\text { permite generar puntos de } \\
\text { interacción en cualquier superficie, se } \\
\text { combina la información producida } \\
\text { anteriormente. }\end{array}$ & $\begin{array}{l}\text { Instalación de } \\
\text { projection mapping } \\
\text { interactivo. }\end{array}$ \\
\hline
\end{tabular}

Como señala Zapata (2015) "la producción de contenidos digitales demanda pasos de producción complejos, dado que la convergencia incluye múltiples soportes y exige una organización informativa pensada en la interacción y la usabilidad para con el usuario"

Se plantean tres niveles de contenidos o capas: la básica y más importante es aquella que puede hacernos una referencia y designación de las principales características de cada área representada en la maqueta, para lo que fue referencial el Prelnventario del IHA, realizada en el marco de un trabajo de extensión de la FADU.

Este nivel permite simultáneamente informar y designar las principales características y la evolución histórica de cada área representada en el modelo a escala a partir del cual se agrupan fotografías, videos y animaciones relacionadas como por ejemplo: Sala de maquinas- Hojalaterías-Cámara de frío y almacenes.
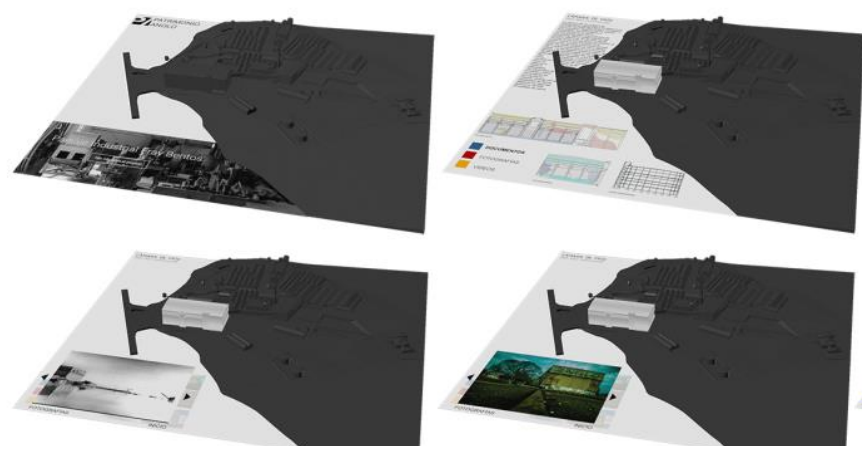

Figure 3. Pruebas de disposición de información y diseño de menús en modelo tridimensional
En resumen, se trabaja sobre el modelo digital generándose capas con información asociada a cada parte de la maqueta.

Para el desarrollo de un segundo nivel de estructuración de contenido fue fundamental el aporte de los guías del museo, así como de docentes de liceo. Este nivel se desarrolló a modo piloto sobre un eje temático en el cual profundizar: por un lado, el recorrido turístico (que abarca casi todo el proceso de producción que se desarrollaba en el Frigorífico Anglo) y por otro la temática de la primera y segunda guerra mundial vinculada al Anglo como para contextualizar el patrimonio involucrado.

En el tercer nivel de contenido dejamos de lado la interacción y se estructura el contenido para adaptarse a un videomapping automático (en loop). A partir de un guion que emula el recorrido turístico se agruparon fotografías documentales y actuales, audiovisuales históricos y actuales de vistas aéreas y se conjugaron en software de edición de
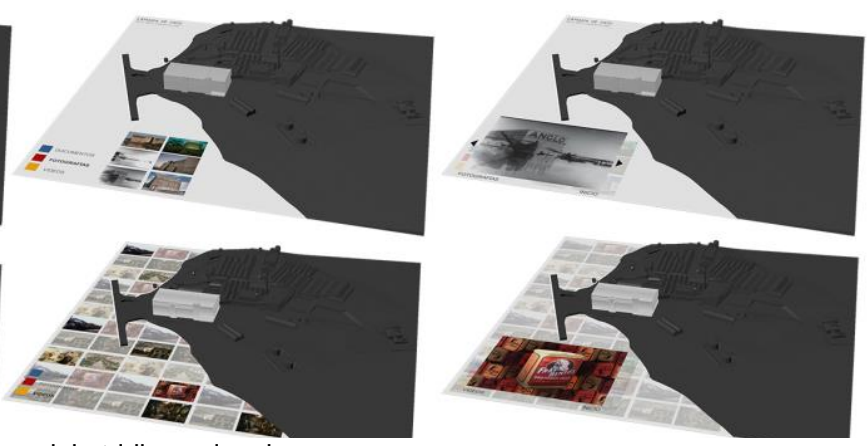
video con el software de mapeo del modelo. Esta parte se configura como espectáculo pasivo, pero con fuerza de impacto visual y sonoro.

3) Reutilización y difusión de contenidos digitales utilizados en la proyección sobre el modelo.

Para esto, se trabajó simultáneamente en la implementación y desarrollo de un repositorio digital utilizando el software de gestión de objetos digitales Omeka. Este utiliza protocolo OAI$\mathrm{PMH}$ (protocolo para trasmisión de contenidos-open access) que permite que los metadatos sean cosechados por otros servicios generando una mayor visibilidad internacional, permitiendo interoperabilidad, intercambio de información y una integración a las redes de fuentes de información cultural nacionales e internacionales.

Por medio de una gran variedad de plugins disponibles para este software, es posible brindar accesibilidad a los contenidos utilizados y producidos para la maqueta para su reutilización posterior por parte de cualquier interesado. También nos brinda herramientas concretas para efectivizar su reutilización tanto sea para generar exposiciones digitales con dichos contenidos, páginas web estáticas con los mismos, facilitar su integración al desarrollo de objetos de aprendizaje en entornos educativos.

En este sentido el repositorio digital ya implementado y en proceso de desarrollo se presenta como una oportunidad para el aprendizaje colaborativo, en tanto permite su difusión y colaborar con ese ciclo de desarrollo de conocimiento y herramientas educativas que estos propician.

\section{Capa 2- Software y hardware utilizado.}

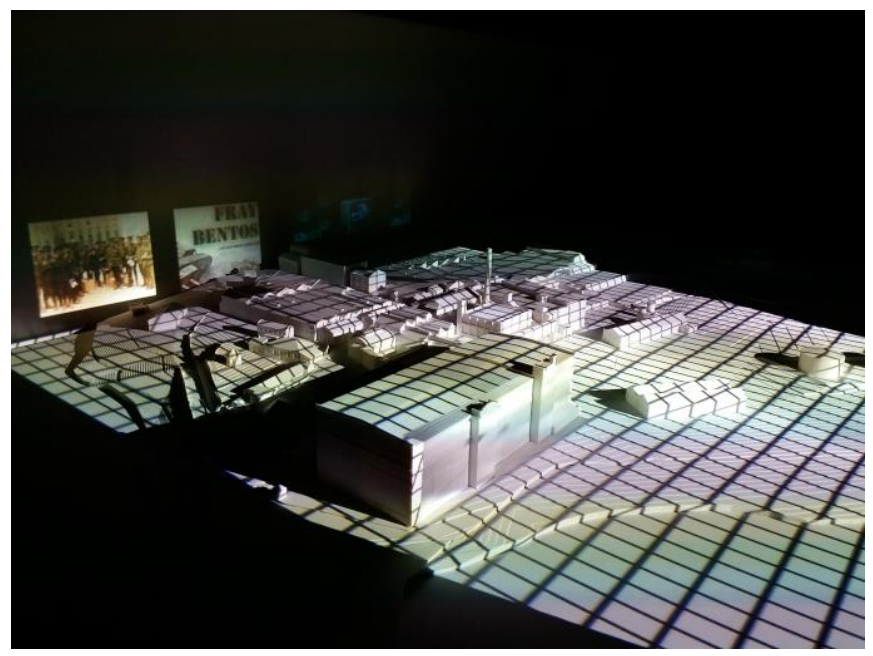

Figura 3. Pruebas de videomapping

En esta capa tenemos dos divisiones metodológicas bastante definidas. Por un lado, el diseño e implementación del videomapping en el modelo, y por otro las posibilidades de interacción por parte de los usuarios con el mismo.

El primer paso para integrar los contenidos digitales, referidos en la sección anterior, al modelo a escala fue el modelo tridimensional digital del mismo. Partiendo de éste se generan clips de video al editarlo en software de diseño 3D como 3ds max. En este software tiene lugar el desarrollo de animaciones de complejidad del modelo tridimensional digital de la maqueta, que son posteriormente renderizadas desde un punto de vista coincidente con la ubicación del proyector en relación a la maqueta real. De esta manera se absorben las deformaciones visuales para que la proyección pueda acoplarse perfectamente a la volumetría del modelo a escala. En el caso estudiado, al tener dos proyectores, se deben hacer dos renderizaciones de video, cada una correspondiente a cada proyector. En el momento de las pruebas en la maqueta misma, se debe lograr la fusión perfecta, o blending de ambas animaciones con una tarjeta gráfica de varias salidas (Matrox) y el software de proyección que da lugar a la calibración definitiva. Con la calibración se ajusta la línea de unión así como la colorimetría o temperatura de color de los proyectores de forma que coincidan.

En paralelo se generan y editan imágenes y videos que compilan los contenidos seleccionados de la etapa anterior. En esta instancia se emplea software de edición raster como Photoshop así como también software de edición de video tales como Adobe Premiere Pro y After Effects para tratar los audiovisuales también producidos en la etapa de producción y edición de contenidos. En el caso de estas animaciones, cobra mayor protagonismo el software de proyección para tratar las deformaciones visuales.

En la instalación propiamente dicha se utilizan dos proyectores de 3000 lumens, entendiendo que el espacio de proyección no era significativo conjuntamente a realizarse en el interior de un edificio permitiendo un control de luz y oscuridad sin exigir luminosidad de proyector.

Conectados mediante una Matrox, hardware-software relacionado al uso de proyectores, una tarjeta gráfica para múltiples pantallas o módulos de expansión gráfica que permite gestionar y conectar más de un proyector a una misma terminal de control de proyección para cubrir la superficie con suficiente intensidad lumínica. Utilizándose generalmente lo que se designa como Matrox (nombre de la empresa canadiense que produce este hardware). Los proyectores fueron colocados a una distancia de 3 metros horizontalmente y a una altura de 2 metros de forma que uno proyecta la imagen sobre la maqueta y el otro sobre la pared. Cabe resaltar que para posicionar las distintas partes fue necesario el diseño de los soportes correspondientes a modo de cubrir la superficie/volumetría de la maqueta. Conjuntamente a estos dispositivos, se integra el sonido mediante el uso de parlantes para cubrir toda el área de la sala en donde estará dispuesta la maqueta.

En lo referente a la interacción, se trabajó conjuntamente con un ingeniero de sistemas debido a la complejidad inherente del proyecto. Este trabajo consistió en la combinación de varios factores: por un lado un sensor de movimiento (cámara Kinect) con un proyector, y por otro el software de proyección con el de interacción que determinó el grado de interactividad con los usuarios. Esta interacción se organizó en una serie de layers de contenidos visuales, sonoros y textuales que se disponen en función de los movimientos de las personas detectados por el sensor.

\section{Consideraciones Finales}

Se puede observar indirectamente y directamente a lo largo 
del proyecto que esta práctica explorada (videomapping o projection mapping), si es realizada teniendo presente algunos conceptos referidos, permite generar lo que podemos entender como un objeto de aprendizaje adaptándose de forma efectiva a fines educativos y de difusión patrimonial. Si bien esto implica cierta complejidad, dificultad y desafíos para su realización, los procesos transitados o planeados transitar nos señalan esta posibilidad.

En lo que refiere a la capa de hardware y software implicados en el proyecto, pudimos observar la importancia determinante de la integración y conjugación de los distintos software de diseño y edición, lo cual constituyó el mayor grado de dificultad al momento de implementar el proyecto. En este sentido, nos recuerdan las observaciones efectuadas por algunos autores como Manovich (2003), sobre esta cualidad de hibridación que caracteriza a estas nuevas prácticas.

Por otro lado, se hace presente que la escala del modelo fue determinante en la planificación del proyecto, observándose que una escala de este tamaño o menor dificulta la realización de animaciones más ricas y detalladas así como el poder trabajar con texturas a un nivel de detalle que pueda ser apreciado a simple vista.

Estando el proyecto en curso y desarrollo (modo piloto), no permite una exposición acabada y concluyente sobre algunos de los aspectos de interés. Falta aún su implementación in situ, lo cual nos permitirá observar y abordar otros aspectos de interés para una investigación de este tipo, como es la observación de la interacción del público con el objeto interactivo.

\section{Debate}

Las oportunidades que brindan las tecnologías de digitalización, visualización avanzada y tendencias asociadas en lo que refiere a la interacción del mundo digital y el mundo real pueden ser novedosas, atractivas y motivantes, pero conjuntamente es relevante que generen procesos significativos. Con procesos significativos nos referimos a procesos de aprendizaje y desarrollo de conocimiento, En el caso tratado, se tiene interés de que los ciudadanos tengan conocimiento sobre el patrimonio Anglo y su relevancia histórica en el contexto nacional e internacional en los dos últimos siglos, los procesos industriales implicados, etc.

Este interés o espíritu de intentar lo significativo, nos reclama para intentar su ejecución y alcanzar en alguna medida parte de estos objetivos, espacios conversacionales teóricosprácticos que integren el diseño digital, la arquitectura, educación, ingeniería y otras especialidades.

En este sentido se entiende relevante exponer las observaciones y descripciones alcanzadas en este tipo de proyectos a las miradas e interpretaciones de distintos actores para fortalecer el debate sobre las temáticas implicadas.

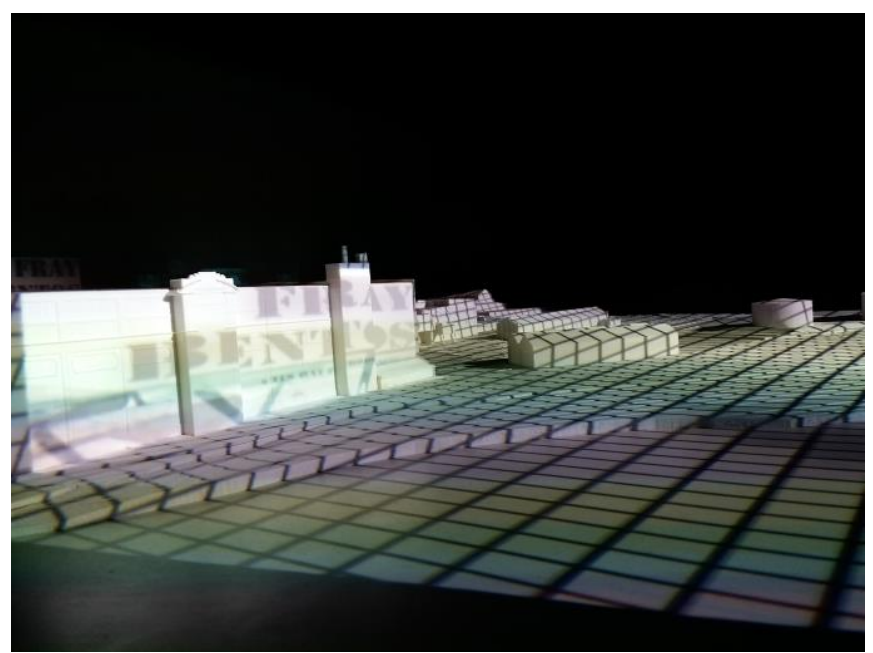

Figura 4. Prueba de mapeado en cámara de frío

\section{Referencias}

Azuma, R.(1997). A survey of augmented reality en Presence: Teleoperators and Virtual Environments n.6 , p.355-385.

Castells, M. (1995). La ciudad informacional. Tecnologías de la Información, reestructuración económica y el proceso urbanoregional. Madrid: Alianza Editorial.

Corboz, A. (2004) "El territorio como palimpsesto". Publicado en "Lo urbano en veinte

autores contemporáneos". Ediciones UPC.

Damino, V.(2006) El frigorífico Anglo y la identidad fraybentina [Tesis]. Montevideo. UdelaR;FCS

Del Moral, M., CERNEA, D.(2005). Diseñando Objetos de Aprendizaje como facilitadores de la construcción del conocimiento. In Proceeding of II Simposio Pluridisciplinar sobre Diseño, Evaluación y Descripción de Contenidos Educativos Reutilizables (SPDECE05.) Barcelona, Spain.

Downes, S. (2010). Design and Reusability of Learning Objects in an Academic Context: A New Economy of Education?. Moncton, Canada: National Research Council Recuperado de http://www.downes.ca/files/milan.doc. Consultado 10/03/2016

Hernández Escobar, O., Pérez Hernández, M. d. P. M., \& Vite Pérez, M. Á. (2015). Ciudades inteligentes: El conocimiento y la tecnología transformando ciudades. Instituto Politécnico Nacional (IPN).

Disponible

en:http://tesis.ipn.mx:8080/xmlui/handle/123456789/14414

IEEE LOM (2002). IEEE 1484.12.1-2002 Standard for Learning Object Metadata.

Recuperado de http://ltsc.ieee.org/wg12. Consultado 10/03/2016

Intendencia Municipal de Rio Negro (2015). Paisaje cultural industrial Fray Bentos. Documento para nominación de Patrimonio MundialUnesco. Fray Bentos. IMM. Pag.275

Karp, C. (2004): "Digital Heritage in Digital Museums", Museum International, n.os 221-222, pp. 45-51.

García, Rafael de Oliveira(2014). Video mapping: um estudo teórico e prático sobre projeção mapeada. . Trabalho de Conclusão de Curso (Bacharel em Comunicação Social - habilitação em Radialismo) - Universidade Estadual Paulista, Faculdade de 
SIGraDi 2016, XX Congreso de la Sociedad Ibero-americana de Gráfica Digital

9-11, November, 2016 - Buenos Aires, Argentina

Arquitetura, Artes e Comunicação Disponible en: <http://hdl.handle.net/11449/119214>.

Garnica,E.; Calderon, J.(2015) Realidad Aumentada y Educación En Revista Ingeniería, Matemática y Ciencia de la Información Vol.2 n.3

Grupo I+D Laboratorio de Visualización Digital Avanzada (2014). Ciudades inteligentes; un palimpsesto digital [on line]. Recuperado de http://www.patrimonioanglo.com/ consultado 10/03/2016

Manso, M. et al. (2011). Contenidos educativos digitales que promueven la integración efectiva de las tecnologías de la información y comunicación. En Digital Education Review. n.19 p.5667

Manovich, Lev (2003). El lenguaje de los nuevos medios de comunicación: la imagen en la era digital. Madrid, Editorial Paidós.

Marzal, M. A.; Calzada Prado, J.; Vianello, M. (2008) Criterios para la evaluación de la usabilidad de los recursos educativos virtuales: un análisis desde la alfabetización en información en Information Research Vol.13, n.4. Recuperado de

http://www.informationr.net/ir/13-4/paper387.html consultado $10 / 03 / 2016$

Prendes Espinosa, C. (2015) Realidad aumentada y educación:

Análisis de experiencias prácticas. En Píxel-Bit. Revista de Medios y

Educación. n. 46 Pag.45-62 Recuperado de

http://dx.doi.org/10.12795/pixelbit.2015.i46.12 consultado
$10 / 03 / 2016$

Rizzo, M. V., \& Artes., U. E. P. ". d. M. F. I. d. (2010). Projeção de vídeo no ambiente urbano: A cidade como tela. São Paulo: [s.n.]. Disponible en VERR.

Universidad de la República. Facultad de Arquitectura (2014) Intenciones Integrales. Montevideo. UdelaR; CSIC. Pag.159

Universidad de la República. Instituto de ingeniería química (2015) Frigorifico Liebig`s: Anglo patrimonio de la humanidad. Montevideo. En Ingeniería Química n.45 p.82-83.

Wilde, E.; Mann, L. (2010) “Open Source Collaboration: New Models for Technology Development in the Museum Community". En: J. Trant and D. Bearman (eds). Museums and the Web 2010:

Proceedings. Toronto: Archives \& Museum Informatics. Disponible en: <http://www.archimuse.com/mw2010/papers/wilde/wilde.html>

Zapata Cárdenas, M. I. U. d. M., Hincapié Montoya, E. M. U. d. M., Díaz León, C. A. U. E., \& Mesías Hoyos, C. U. d. M. (2015). Generación de contenidos digitales para la reactivación del patrimonio arquitectónico. Estudio de caso: Plaza de mercado de techo cubierto de Guayaquil, Medellín. Universidad de Medellín. 